\title{
Research report \\ Neurotoxic lesions of the dorsal hippocampus and Pavlovian fear conditioning in rats
}

\author{
Stephen Maren ${ }^{\mathrm{a}, *}$, Gal Aharonov ${ }^{\mathrm{b}}$, Michael S. Fanselow ${ }^{\mathrm{b}}$ \\ a Department of Psychology and Neuroscience Program, University of Michigan, 525 East University Avenue, Ann Arbor, MI 48109-1109, USA \\ b Department of Psychology, University of California, 405 Hilgard Avenue, Los Angeles, CA 90095-1563, USA
}

Received 10 May 1997; accepted 19 May 1997

\begin{abstract}
Electrolytic lesions of the dorsal hippocampus (DH) produce deficits in both the acquisition and expression of conditional fear to contextual stimuli in rats. To assess whether damage to DH neurons is responsible for these deficits, we performed three experiments to examine the effects of neurotoxic $N$-methyl-D-aspartate (NMDA) lesions of the DH on the acquisition and expression of fear conditioning. Fear conditioning consisted of the delivery of signaled or unsignaled footshocks in a novel conditioning chamber and freezing served as the measure of conditional fear. In Experiment 1, posttraining DH lesions produced severe retrograde deficits in context fear when made either 1 or 28, but not 100, days following training. Pretraining DH lesions made 1 week before training did not affect contextual fear conditioning. Tone fear was impaired by DH lesions at all training-to-lesion intervals. In Experiment 2, posttraining (1 day), but not pretraining (1 week), DH lesions produced substantial deficits in context fear using an unsignaled shock procedure. In Experiment 3, pretraining electrolytic DH lesions produced modest deficits in context fear using the same signaled and unsignaled shock procedures used in Experiments 1 and 2, respectively. Electrolytic, but not neurotoxic, lesions also increased pre-shock locomotor activity. Collectively, this pattern of results reveals that neurons in the DH are not required for the acquisition of context fear, but have a critical and time-limited role in the expression of context fear. The normal acquisition and expression of context fear in rats with neurotoxic DH lesions made before training may be mediated by conditioning to unimodal cues in the context, a process that may rely less on the hippocampal memory system. (C) 1997 Elsevier Science B.V.
\end{abstract}

Keywords: Amnesia; Fear conditioning; Context; Hippocampal formation; Anterograde; Retrograde; Lesion; Locomotor activity; Freezing; Rat

\section{Introduction}

In Pavlovian conditioning experiments, it is typical that relationships are arranged between discrete conditional stimuli (CSs), such as lights or tones and unconditional stimuli (USs), such as food or footshocks. Although much of the learning in these situations reflects an association between the discrete CS and US,

\footnotetext{
* Corresponding author. Tel.: + 1313 9366532; fax: + 1313 7637480; e-mail: maren@umich.edu
}

there is substantial evidence that the US also becomes associated with the training environment, which is commonly referred to as the training context. Thus, in Pavlovian fear conditioning, rats trained with tonefootshock pairings exhibit substantial conditional fear (measured as freezing behavior) to both the tone CS and the context of the conditioning chamber in which training occurred. Because tone CSs are relatively simple (unimodal, discrete) and context CSs are complex (multimodal, diffuse), it is typically assumed that rats use an elemental strategy to acquire tone fear and a 
configural strategy to acquire context fear. However, we assume that fear to tone or context CSs can be acquired using either elemental or configural strategies. By this view, both tone and context CSs are assumed to be composed of individual elements that can enter into association with a US either individually (elemental strategy) or as a codified group (configural strategy, e.g. [8]).

Concerning the neural substrates of fear conditioning, recent studies indicate that fear conditioning to both contextual and discrete stimuli requires neurons in the amygdala $[4,15,16,18]$. Furthermore, recent studies indicate that electrolytic lesions of the dorsal hippocampus (DH) produce substantial and selective deficits in both the acquisition and expression of context fear, measured as freezing in the training apparatus. Specifically, conditional freezing to contextual CSs is disrupted by electrolytic DH lesions made either before $[14,19,25,26,40]$ or shortly after [13] fear conditioning. Impairments in context fear occur when USs are unsignaled $[14,19,26]$ or signaled by a tone CS $[13,25,26]$. Deficits in context fear have also been reported following electrolytic lesions of the fimbria/ fornix or entorhinal cortex $[17,19,27]$. In contrast, fear conditioning to discrete CSs, such as a tone, is typically not affected by hippocampal formation lesions [13,25$27,17]$. Based on these data, it has been proposed that the $\mathrm{DH}$ has a selective role in fear conditioning to contextual stimuli, but is not required for conditioning to discrete stimuli. If rats tend to use a configural strategy to learn about contexts and an elemental strategy to learn about discrete CSs, then the findings with hippocampal lesions appear consistent with the proposed role of the hippocampal formation in spatial and configural learning [24,30,34].

Although many experiments implicate the hippocampal formation, particularly the $\mathrm{DH}$, in the mechanisms underlying contextual fear conditioning, it is not clear to what extent lesion-induced deficits in conditional freezing reflect damage to neurons in the hippocampus or damage to axonal fibers of passage. Therefore, in the present study, we examined the effects of neurotoxic hippocampal lesions on fear conditioning in rats using freezing as a measure of conditional fear. Neurotoxic lesions were made in the DH using $N$-methyl-D-aspartate (NMDA), a potent excitotoxin that produces neuronal lesions in both the hippocampus proper and dentate gyrus, but spares axonal fibers of passage. NMDA infusions into the hippocampal formation produce minimal extra-hippocampal damage [10]. To assess the involvement of DH neurons in the acquisition and expression of fear conditioning, we examined the effects of both pre and posttraining neurotoxic DH lesions on contextual fear conditioning established with either signaled or unsignaled shock procedures. Fear conditioning to the tone CS was assessed in the signaled shock procedures.
In contrast to previous studies using electrolytic lesions, the results of the present experiments reveal that $\mathrm{DH}$ neurons are not required for learning context fear, but are nonetheless required for expressing context fear memories. To account for these data we elaborate a conceptual model which holds that context fear can be acquired using one of two strategies: A hippocampusdependent configural strategy or a hippocampus-independent elemental strategy. We suggest that normal acquisition and expression of context fear in rats with neurotoxic DH lesions is mediated by conditioning to unimodal cues in the context, a process that relies less on the hippocampal memory system. To account for the impairing effects of electrolytic $\mathrm{DH}$ lesions on the acquisition of context fear, the model posits that fibers of passage in the DH mediate the exploratory behavior that is required for processing contextual stimuli. Because electrolytic or neurotoxic DH lesions produce deficits in freezing to a tone CS, we further suggest that fear to discrete CSs requires the hippocampus under some circumstances.

\section{Experiment 1: Conditional fear following signaled footshock in rats with neurotoxic DH lesions}

Previous work indicates that electrolytic DH lesions produce deficits in both the acquisition and expression of contextual fear conditioning, but do not affect conditioning to acoustic CSs $[13,25,26]$. Moreover, deficits in the expression of contextual fear conditioning after posttraining DH lesions have been reported to be timelimited, decreasing in magnitude 2 weeks after conditioning and disappearing 1 month after conditioning [13]. It is not known, however, if these deficits are due to damage to neurons in the dorsal hippocampus, axonal fibers of passage, or both. This question was addressed in the following experiment by examining the impact of pre- and posttraining neurotoxic DH lesions on Pavlovian fear conditioning to contextual and acoustic CSs. DH lesions were made with NMDA either 7 days before or 1, 28, or 100 days after Pavlovian fear conditioning. Fear conditioning was established using tone-footshock pairings, a 'signaled' shock procedure.

\subsection{Materials and methods}

Subjects. The subjects were 164 adult male LongEvans rats $(300-500 \mathrm{~g})$ born and reared in the Department of Psychology vivarium at the University of California, Los Angeles. After weaning, the rats were group housed in same sex cohorts. At the beginning of the experiment, the rats were individually housed in standard stainless-steel hanging cages on a 14:10-h light-dark cycle (lights on at 07:00 h) and had free 
access to food and tap water. After individual housing, the rats were handled daily (10-20-s per rat) for 5 days to acclimate them to the experimenter.

Surgery. The rats were anesthetized with an intraperitoneal injection of Nembutal (sodium pentobarbital, $65-\mathrm{mg} / \mathrm{kg}$ body weight) and mounted in a stereotaxic apparatus (David Kopf Instruments, Tujunga, CA). The scalp was incised and retracted and head position was adjusted to place bregma and lambda in the same horizontal plane. Small burr holes (2-mm diameter) were drilled bilaterally in the skull for the placement of 28-gauge cannulae in the dorsal hippocampus (DH). Separate infusions were made in the rostral DH (2.8$\mathrm{mm}$ posterior to bregma and $1.6-\mathrm{mm}$ lateral to the midline) and the caudal $\mathrm{DH}$ (4.2- $\mathrm{mm}$ posterior to bregma and 2.6-mm lateral to the midline). A $10 \mu 1$ Hamilton syringe was mounted in an infusion pump (Harvard Apparatus, South Natick, MA) and connected to the injection cannula with polyethylene tubing. NMDA $(20-\mu \mathrm{g} / \mu 1$; Sigma, St. Louis, MO) in 100-mM phosphate-buffered saline (PBS, pH 7.4) was infused $(0.1-\mu 1 / \mathrm{min} ; 0.4 \mu \mathrm{l}) 3.3 \mathrm{~mm}$ ventral to brain surface for the rostral penetrations and $3.0 \mathrm{~mm}$ ventral to brain surface for the caudal penetrations. The rostral infusions always preceded the caudal infusions and 5 min were allowed after each infusion for diffusion of the drug. Sham groups received the same surgical procedures, but did not receive cannula implantation or infusions. Following surgery, the incision was closed with stainless steel wound clips and the rats were allowed to recover on a heating pad before returning to their home cage. Rats with NMDA infusion into the DH typically showed behavioral signs of the infusions during recovery from anesthesia. This was manifest as a long duration to recover the righting reflex, shivering and brief bouts of running once the righting reflex was recovered. However, the rats never exhibited tonicclonic seizures, nor did the rats exhibit signs of seizure activity following recovery from surgery. A total of three rats were excluded from the data analysis due to death following surgery.

Behavioral apparatus. A total of eight identical observation chambers $(28 \times 21 \times 22 \mathrm{~cm}$; Lafayette Instrument, North Lafayette, IN) were used for both conditioning and context fear testing. The chambers were constructed from aluminum (sidewalls) and Plexiglas (rear wall, ceiling and hinged front door). The chambers were situated in chests located in a brightly lit and isolated room. A videocamera placed in front of the observation chambers allowed each subjects' behavior to be observed and recorded by an experimenter in an adjacent room. The floor of each chamber consisted of 18 stainless steel rods (4-mm diameter) spaced $1.5 \mathrm{~cm}$ apart (center-to-center). The rods were wired to a shock generator and scrambler (Lafayette) for the delivery of footshock USs. A speaker located on one wall of the chamber permitted the delivery of tone CSs. The chambers were cleaned with a $5 \%$ ammonium hydroxide solution and stainless steel pans containing a thin film of the same solution were placed underneath the grid floors before rats were placed inside. Background noise (70 dB, A-scale) was supplied by ventilation fans in each chest and adjacent shock scramblers.

An additional set of eight observation chambers $(28 \times 21 \times 22 \mathrm{~cm}$; Lafayette $)$ located in an adjacent room were used for testing conditional fear to the tone CS. These chambers were constructed as described above and were situated in chests located in a quiet, dimly lit, and isolated room. A number of modifications were made to this testing environment to minimize its similarity to the training context. Ambient light in the room was provided by a single lamp equipped with a 15-W red light bulb. The floor of each chamber consisted of 17 vertically staggered stainless steel rods (4-mm diameter) spaced $1.5 \mathrm{~cm}$ apart (center-to-center) and the outside of the rear wall of the chambers was covered with white construction paper. In addition, a white, opaque Plexiglas tent was inserted into each chamber so that the apex of the tent contacted the roof of the chamber and the open base of the tent fit into the bottom corners of the chamber. A speaker located on one wall of the chamber permitted the delivery of acoustic CSs. Background noise (70 dB, A-scale) was supplied by a white noise generator. The chambers were cleaned with a $1 \%$ acetic acid solution and stainless steel pans containing a thin film of the same solution were placed underneath the grid floors before rats were placed inside. A videocamera placed in front of the observation chambers allowed each subjects' behavior to be observed and recorded by an experimenter in an adjacent room.

Procedure. The rats were randomly assigned to a $2 \times 4$ (lesion $\times$ training-to-lesion interval) design. For this design, rats received either NMDA lesions in the DH or sham surgery 7 days before (anterograde, $n=$ $22 /$ group) or 1 ( $n=25 /$ group), 28 ( $n=20 /$ group), or 100 ( $n=15 /$ group) days after (retrograde) Pavlovian fear conditioning using signaled footshocks. On the conditioning day, the rats were transported to the laboratory and placed in the conditioning chambers in squads of eight rats; the chamber position was counterbalanced for each squad and group. The rats received three tone (10-s, 90-dB, 2-kHz)-footshock (2-s, 1.0-mA) pairings (74-s intertrial interval) $3 \mathrm{~min}$ after being placed in the chambers. The rats were returned to their home cages $64 \mathrm{~s}$ after the final shock.

For rats that received surgery prior to training (anterograde groups), fear conditioning on the training day was assessed by measuring freezing during the 64-s period before the first tone-shock trial and the 64-s periods following each of the three tone-shock trials (i.e. postshock freezing). Briefly, an observer who was 

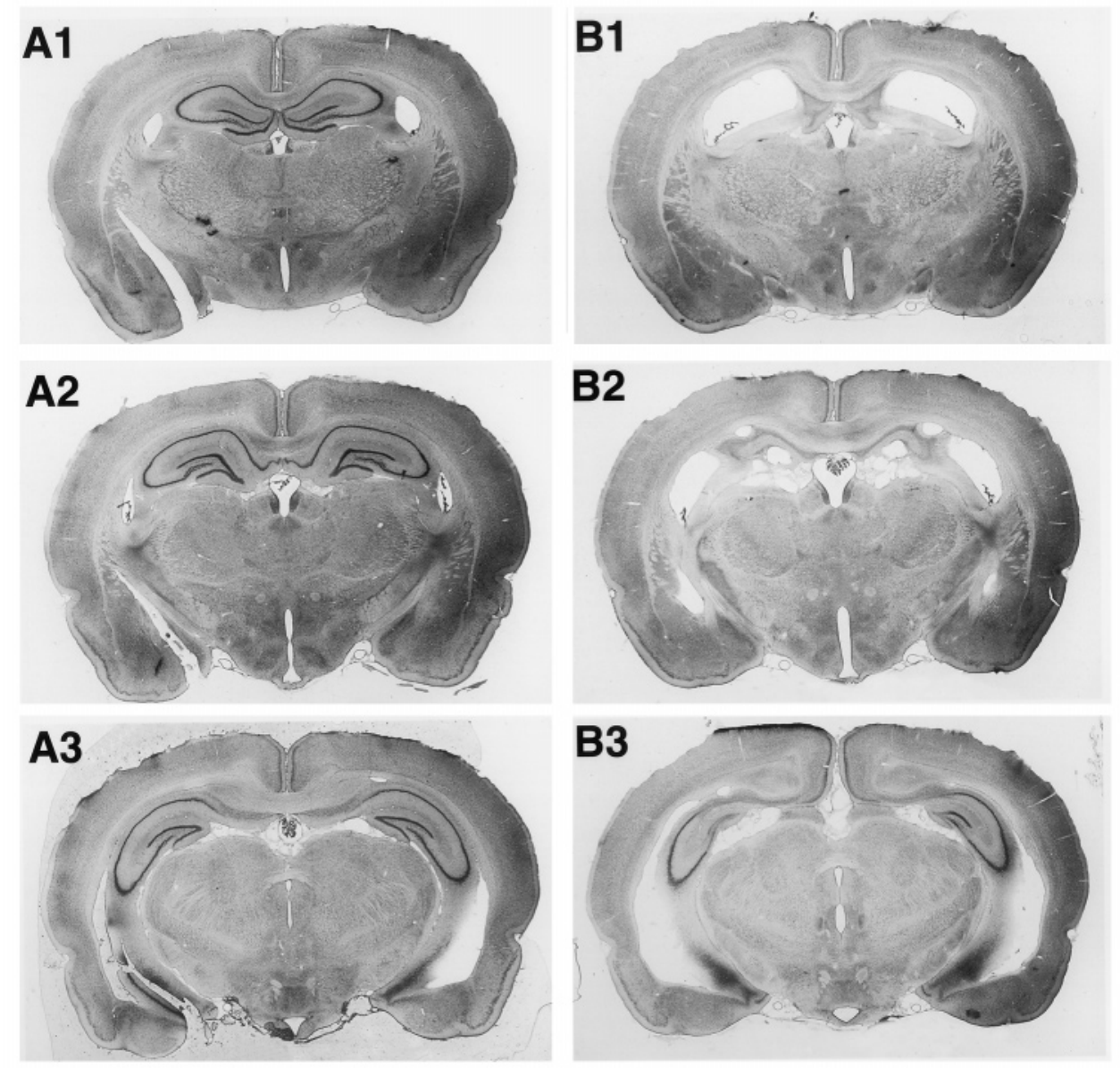

Fig. 1. Photomicrographs showing thionin-stained coronal brain sections at three rostral-caudal levels (1-3) from (A) a rat receiving sham surgery and (B) a rat receiving $N$-methyl-D-aspartate (NMDA) infusion into the dorsal hippocampus. The lesion depicted in (B) is representative of that observed in all of the rats receiving intra-hippocampal NMDA infusion.

blind to the experimental conditions scored each rat for freezing (behavioral immobility except for movement necessitated by respiration) every $8 \mathrm{~s}$ for a total of eight observations per animal. Fear conditioning to the context was assessed $24 \mathrm{~h}$ after training by returning the rats to the conditioning chambers and scoring freezing during an 8-min 32-s extinction test, yielding a total of 64 observations per rat. On the following day, fear conditioning to the tone was assessed in an 'off-baseline' extinction test, that is, the tone test was conducted in a context different from the training context. The rats were placed in observation chambers that were distinct from those used during conditioning and after 2 min, presented with an 8-min 32-s tone. For rats receiving surgery after training, the context and tone tests were performed 1 week following surgery to allow for recovery.

Histology. Histological verification of lesion location was performed after behavioral testing. Rats were perfused across the heart with $0.9 \%$ saline followed by $10 \%$ formalin. After extraction from the skull, the brains were post-fixed in $10 \%$ formalin for 2 days and $10 \%$ formalin $/ 30 \%$ sucrose until sectioning. Coronal sections (50 $\mu \mathrm{m}$ thick, taken every $200 \mu \mathrm{m}$ ) were cut on a cryostat $\left(-16^{\circ} \mathrm{C}\right)$ and wet-mounted on glass microscope slides with $70 \%$ ethanol. After drying, the sections were stained with $0.25 \%$ thionin to visualize neuronal cell bodies. Lesions were verified by visual inspection of the stained brain sections.

Data analysis. For each test period, the freezing data were transformed to a percentage of total observations, a probability estimate that is amenable to analysis with parametric statistics. These probability estimates of freezing were analyzed using analysis of variance (ANOVA). Planned comparisons in the form of Fisher LSD tests were performed following a significant omnibus $F$-ratio. All data are represented as means \pm the standard errors of the means (S.E.M.).

\subsection{Results and discussion}

Histology. Photomicrographs of coronal brain sections from representative rats in the sham and lesion groups are shown in Fig. 1. All of the rats in the DH 
groups had complete, bilateral lesions of the dorsal hippocampal formation, including pyramidal neurons in hippocampal areas CA1 and CA3 and granular neurons in the dentate gyrus. The lesions were remarkably consistent among rats in the lesion groups, and are generally similar to the lesion shown in Fig. 1. The NMDA infusions caused a gradient of tissue damage, ranging from extreme cell loss at the locus of the cannula tip to relatively minor cell loss at the caudal borders of the infusion. Typically, the lesions included the most rostral aspect of the dorsal subiculum, but the ventral hippocampus and ventral subiculum were spared. We estimate that on average $40 \%$ of the total volume of the hippocampus was destroyed by the lesions. Inspection of the stained tissue sections did not reveal damage to either extrahippocampal structures or white matter tracts (alveus, fimbria, or corpus callosum) in the vicinity of the lesion in any of the rats. This is consistent with the pattern of damage produced by intrahippocampal infusions of ibotenate, which Jarrard and Meldrum [10] have reported is comparable to that produced by intrahippocampal NMDA infusion. Importantly, there was no systematic variation in either the size or nature of the DH lesions across the different training-to-lesion intervals. In general, our neurotoxic DH lesions were comparable in size to those obtained with electrolysis in other studies [19], although the latter destroyed axonal tracts passing through the DH.

Behavior. To assess the impact of neurotoxic DH lesions on the acquisition of a short-term memory for conditional freezing, we assessed postshock freezing on the conditioning day for rats in the anterograde groups. As shown in Fig. 2, pretraining neurotoxic DH lesions did not attenuate postshock freezing on the conditioning day. This observation was confirmed in a two-way ANOVA with variables of lesion ( $\mathrm{SH}$ and $\mathrm{DH}$ ) and training trial (baseline and one, two and three trials) by a nonsignificant main effect of lesion $[F(1,42)=1.1, P=0.29]$ and a nonsignificant interaction of lesion and training trial $[F(3,126)=2.2, P=$ 0.09]. A significant main effect of training trial $[F(3,126)=122.0, P<0.0001]$ indicated that rats in both the sham and DH lesion groups acquired conditional freezing on the conditioning day. Although some reports have observed normal postshock freezing in rats with electrolytic DH lesions [14], it is more typical that electrolytic DH lesions attenuate postshock freezing $[19,40]$. Therefore, these results suggest that neurotoxic and electrolytic DH lesions differentially affect the acquisition of short-term conditional freezing.

As shown in Fig. 3, however, neurotoxic lesions of the DH produced marked deficits in conditional freezing to both contextual and tone CSs during the posttraining extinction tests, a measure of long-term memory. For statistical analysis, the data were collapsed across the eight 64-s blocks of the extinction test and separate analyses were performed for tone and context freezing. For context freezing, a two-way ANOVA with factors of lesion ( $\mathrm{SH}$ and $\mathrm{DH})$ and training-to-lesion interval ( $-7,1,28$ and 100 days) revealed significant main effects of group $[F(1,155)=$ 65.5, $\quad P<0.0001], \quad$ training-to-lesion interval $[F(3,155)=12.7, P<0.0001]$ and a significant group $\times$ training-to-lesion interval interaction $[F(3,155)=6.4$, $P<0.001]$. Planned comparisons $(P<0.05)$ indicated that pretraining DH lesions did not produce deficits in contextual freezing, whereas posttraining DH lesions made either 1,28 , or 100 days following training produced significant deficits in freezing relative to sham controls. The retrograde deficit was not uniform across the training-to-lesion interval, that is, freezing in rats that received DH lesions 100 days following training was significantly greater than that in rats receiving lesions either 1 or 28 days following training; freezing in the sham rats did not vary across the training-to-lesion interval. Thus, the retrograde deficit was temporally graded or time-limited. However, freezing in rats with DH lesions made 100 days after training was still lower than that in sham rats. Freezing in the sham rats was stable over the training-to-lesion intervals.

In contrast to context freezing, freezing to the tone CS was attenuated by both pre- and posttraining DH lesions (none of the rats froze in the pre-tone period). This was confirmed by significant main effects of lesion

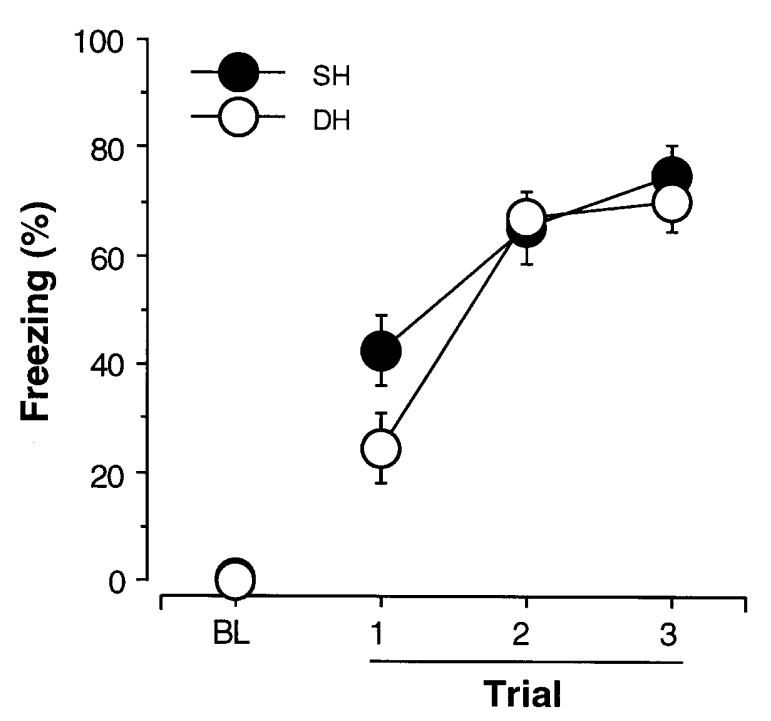

Fig. 2. Pretraining neurotoxic lesions of the dorsal hippocampus and postshock freezing. Mean $( \pm$ S.E.M. $)$ percentage of freezing on the conditioning day for rats in the sham ( $\mathrm{SH}$, filled circles) and dorsal hippocampus (DH, open circles) groups. Freezing was scored during 64-s intervals before (baseline or BL) and after each of the three tone-shock trials. Rats with neurotoxic DH lesions showed equivalent levels of postshock freezing compared to rats receiving sham surgery. 

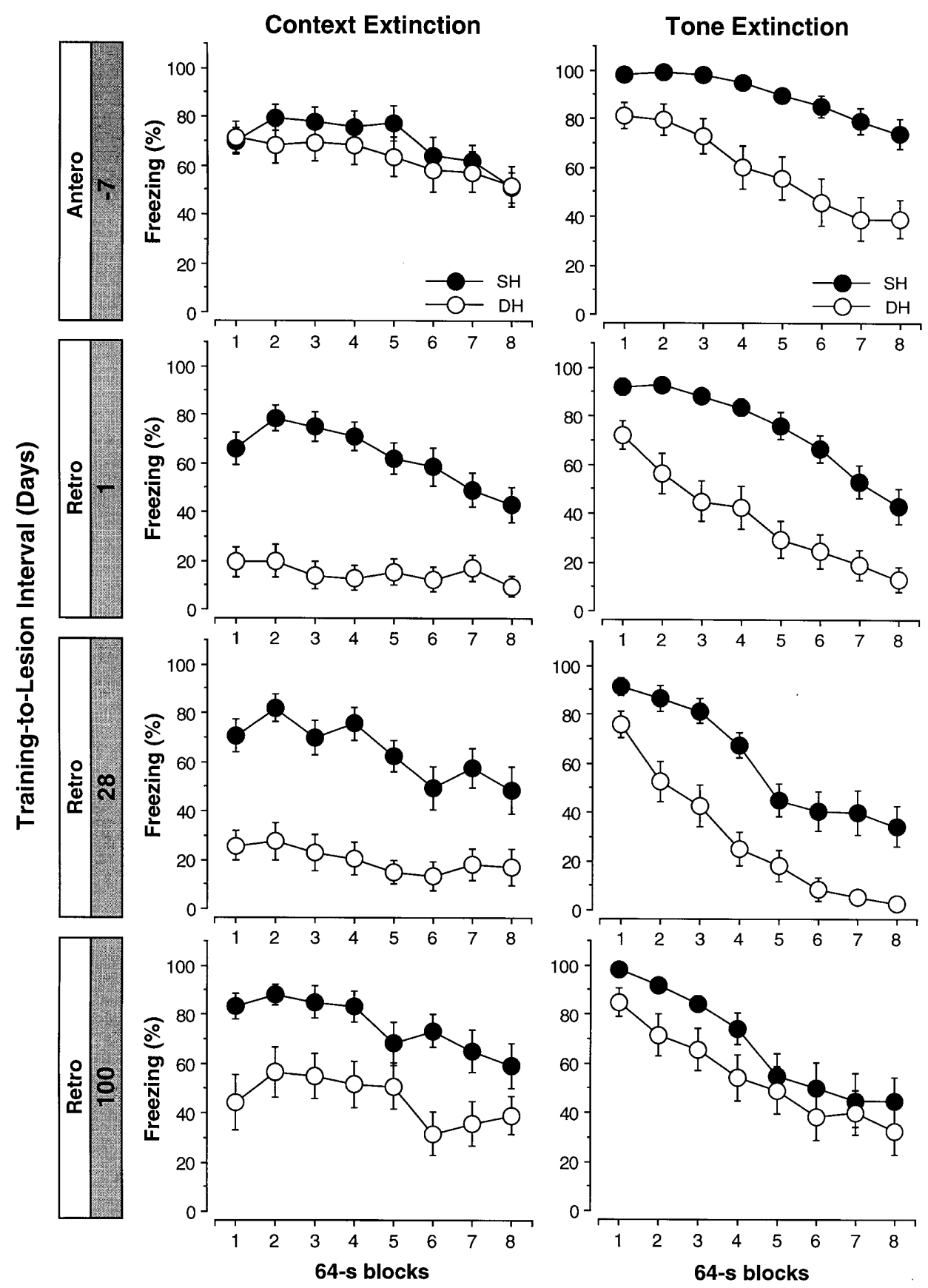

Fig. 3. Pretraining and posttraining neurotoxic lesions of the dorsal hippocampus and freezing during context and tone extinction tests. Mean ( \pm S.E.M.) percentage of freezing during the context (leftmost column) or tone (rightmost column) extinction test in rats receiving surgery either 7 days before (anterograde) or 1, 28, or 100 days after (retrograde) fear conditioning for sham (SH, filled circles) and dorsal hippocampus (DH, open circles) groups. Extinction testing commenced 1 day after training for the anterograde groups and 1 week following recovery from surgery for each of the retrograde groups. The tone extinction test was run one day following the context extinction test. Neurotoxic DH lesions produced deficits in conditional freezing to both the context and tone, and these deficits varied across the training-to-lesion interval for contextual, but not tone fear.

$[F(1,155)=53.2, P<0.0001]$ and training-to-lesion interval $[F(3,155)=10.8, P<0.0001]$ in the ANOVA. However, the tone freezing deficit did not appear to vary as a function of training-to-lesion interval as indicated by a nonsignificant interaction of lesion and training-to-lesion interval $[F(3,155)=1.5, \quad P=0.21]$. Nonetheless, planned comparisons indicated that freez- ing to the tone in rats receiving DH lesions 100 days following training was significantly greater than that in rats receiving lesions either 1 or 28 days following conditioning, but was not significantly different from rats receiving sham surgery 100 days after training. This suggests a trend for the tone freezing deficit to be time-limited. 

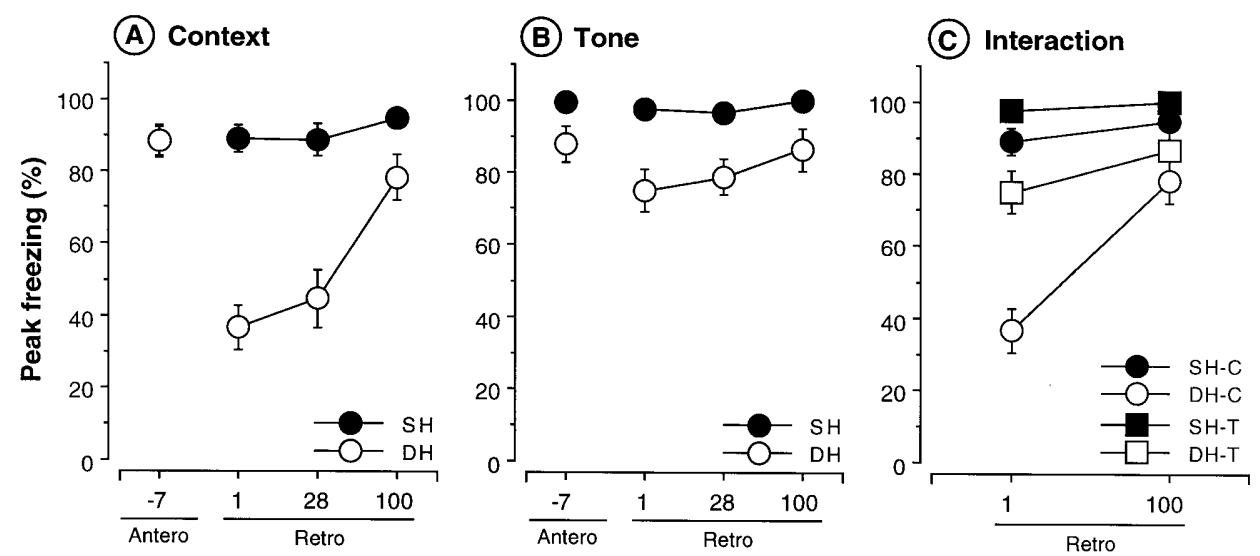

Training-to-lesion interval (days)

Fig. 4. Pretraining and posttraining neurotoxic lesions of the dorsal hippocampus and peak conditional freezing. Mean ( \pm S.E.M.) percentage of peak freezing during the (A) context and (B) tone extinction tests in rats receiving surgery either 7 days before (anterograde) or 1, 28, or 100 days after (retrograde) fear conditioning for sham ( $\mathrm{SH}$, filled circles) and dorsal hippocampus ( $\mathrm{DH}$, open circles) groups. Values represent means derived from the first 4 min of the extinction data shown in Fig. 3. Neurotoxic DH lesions produced deficits in conditional freezing to both the context and tone and these deficits varied across the training-to-lesion interval for contextual, but not tone fear. (C) A subset of the data shown in (A) and (B) that illustrates the interaction between lesion, conditional stimulus and training-to-lesion interval.

For a direct comparison of freezing to tone and context CSs across the training-to-lesion interval, we analyzed peak freezing in sham rats and rats with $\mathrm{DH}$ lesions. Peak freezing consisted of the maximum level of freezing obtained by each subject in any 64-s block of the tone or context extinction tests. This measure provided an estimation of CR magnitude that was not diluted by the substantial extinction accrued during testing. As shown in Fig. 4(a) and (b), the pattern of deficits in peak freezing in rats with DH lesions was nearly identical to that revealed in the analysis of the 8-min 32-s means. Thus, only the analysis of context freezing revealed a significant group $\times$ training-to-lesion-interval interaction [context: $F(3,155)=11.2, P<$ 0.0001 ; tone: $F(3,155)=0.8, P=0.48]$. The critical analysis comparing peak freezing to tone and context across the short (1 day) and long (100 day) training-tolesion-intervals is shown in Fig. 4(c). A three-way ANOVA with factors of lesion ( $\mathrm{SH}$ and $\mathrm{DH}$ ), $\mathrm{CS}$ (tone and context) and training-to-lesion interval (1 and 100 days) revealed a significant three-way interaction $[F(1,76)=5.5, P<0.05)$. This reveals that the time-limited deficit in rats with $\mathrm{DH}$ lesions is significantly greater to contexts than to tones.

Collectively, these results are consistent with the time-limited retrograde impairments in contextual fear conditioning that have been reported following electrolytic DH lesions [13]. These results indicate that neurons in the DH have a time-limited role in expressing context fear acquired by intact rats, which is consistent with other findings from various animal models $[6,7,12,38,39,41]$ and humans [28,32] that the hippocampal system has a time-limited role in memory processes.
Nonetheless, a number of results suggest that neurotoxic and electrolytic DH lesions have differential effects on fear conditioning. First, acquisition of context fear in rats with neurotoxic DH lesions was not impaired. This suggests that damage to axonal fibers of passage, particularly those running in the fimbria/ fornix, may be responsible for deficits in the acquisition of context fear in rats with electrolytic DH lesions. Moreover, the absence of an anterograde impairment in context fear in rats with neurotoxic DH lesions suggests that context fear can be learned with a non-hippocampal strategy. Second, neurotoxic DH lesions produced significant impairments in tone freezing. While this has not been reported after electrolytic DH lesions $[13,25,26]$, we report in Experiment 3 (Section 4) that tone freezing generated by the conditioning parameters used in the present study is affected by electrolytic DH lesions. Lesion-induced tone fear impairments in the present experiments may be related to the shift from the short duration tone $(10 \mathrm{~s})$ in training to a long duration tone ( $8 \mathrm{~min})$ during testing. Indeed, rats with neurotoxic DH lesions do not differ from shams during the first $10 \mathrm{~s}$ of the tone extinction test. The deficit beyond the first $10 \mathrm{~s}$ of the tone test may be due to a failure of rats with hippocampal lesions to generalize from short to long tones or more rapid extinction of tone fear in these rats. And lastly, the time course of the retrograde gradient observed in the present study was considerably different than that reported in an earlier study using electrolytic DH lesions [13]. The comparatively long retrograde gradient observed in the present experiment (100 days) may be due to several factors including the sex of the rats, the type or extent of the DH lesions, or the nature or strength of the conditioning. For instance, 
it is possible that the large number of conditioning trials (15 trials compared to three trials in the present experiments) used by Kim and Fanselow [13] facilitated consolidation of the context fear memory in extrahippocampal structures, thereby shortening the retrograde gradient.

It should also be pointed out that the pattern of results we have observed following neurotoxic DH lesions is quite different from that reported by Selden et al. [31] who found decreased conditioning to context and normal conditioning to tones in rats with ibotenate hippocampal lesions. The reason for this disparity is not known, but may reflect the different procedures used to assess conditional fear (place preference versus freezing) or the larger neurotoxic hippocampal lesions employed in the experiments of Selden et al. [31].

\section{Experiment 2: Conditional fear following unsignaled footshock in rats with neurotoxic DH lesions}

Several studies have reported that the electrolytic DH lesions disrupt the acquisition and expression of contextual freezing not only when footshocks are signaled $[13,25,26]$, but also when they are unsignaled $[14,19,40]$. It is of considerable interest to determine whether deficits in unsignaled contextual fear conditioning produced by electrolytic DH lesions are mediated by specific damage to DH neurons. Therefore, the following experiment examined the impact of neurotoxic DH lesions made either 1 week before or 1 day after contextual fear conditioning established with an unsignaled shock procedure. In this experiment, rats in both the pretraining and posttraining groups were tested 1 week following training, because the short training-to-testing interval in the pretraining groups in Experiment 1 may have masked an anterograde deficit manifest as more rapid forgetting of conditioning.

\subsection{Materials and methods}

Subjects, surgery and behavioral apparatus. The subjects were 60 adult male Long-Evans rats $(300-500 \mathrm{~g})$ obtained and housed as described in Experiment 1 (Section 2). Furthermore, the surgery and the conditioning chambers were identical to those described in Experiment 1. However, only the chambers used for conditioning and context fear testing in Experiment 1 were used in the present experiment.

Procedure. The rats were randomly assigned to a $2 \times 2$ (lesion $\times$ training-to-lesion interval; $n=15 /$ group) design. For this design, rats received either NMDA lesions in the DH or sham surgery 7 days before (anterograde) or 1 day after (retrograde) Pavlovian fear conditioning using unsignaled footshocks. All of the procedures for fear conditioning were identical to those described in Experiment 1, except that the footshocks were not signaled by tones. Context fear testing for all groups of rats was conducted during a 10-min extinction test 1 week following training.

Histology and data analysis. Histological verification of lesion location and data analysis were performed as described for Experiment 1.

\subsection{Results and discussion}

Histology. The nature and extent of the NMDA lesions in this experiment were virtually identical to those described in Experiment 1. All rats had complete, bilateral lesions of the $\mathrm{DH}$ with sparing of axonal fibers of passage.

Behavior. Postshock freezing on the conditioning day for rats in each group is shown in Fig. 5(a). Rats in each group acquired conditional freezing during training and the groups did not differ from one another in this regard. This impression was confirmed in a threeway ANOVA with variables of lesion ( $\mathrm{SH}$ and $\mathrm{DH})$, training-to-lesion interval (pre- and posttraining) and training trial (baseline and one, two and three trials) by a significant main effect of trial $[F(3,168)=115.1, P<$ $0.0001]$ and a nonsignificant effect of lesion $[F(1,56)=$ $0.1, \quad P=0.81]$ or lesion $\times$ training-to-lesion interval interaction $[F(1,56)=1.1, P=0.29]$. Thus, as in Experiment 1, pretraining neurotoxic DH lesions did not affect the acquisition of postshock freezing during contextual fear conditioning.

Freezing during the $10 \mathrm{~min}$ context extinction test is shown in Fig. 5(b). As in Experiment 1, neurotoxic DH lesions produced an impairment in conditional freezing to contextual cues when made 1 day after, but not 7 days before, fear conditioning. This was confirmed in a three-way ANOVA with variables of lesion ( $\mathrm{SH}$ and
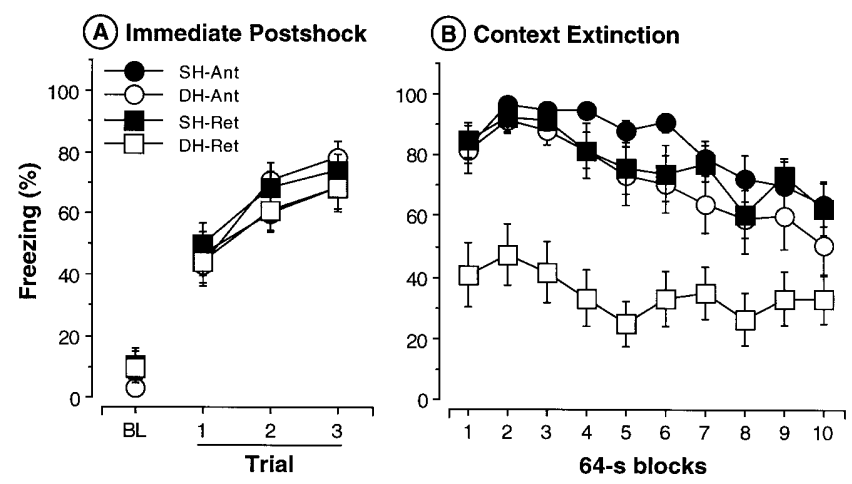

Fig. 5. Pretraining and posttraining neurotoxic lesions of the dorsal hippocampus and conditional freezing following unsignaled shock. (A) Mean ( \pm S.E.M.) percentage of freezing on the conditioning day for rats in the sham (SH, filled circles) and dorsal hippocampus (DH, open circles) groups and (B) mean ( \pm S.E.M.) percentage of freezing during the context extinction test for rats in the sham $(\mathrm{SH}$, filled circles) and dorsal hippocampus (DH, open circles) groups. Retrograde, but not anterograde, DH lesions impaired contextual freezing. 
$\mathrm{DH})$, training-to-lesion interval (pre- and posttraining), and 64-s block (ten blocks) by a significant main effect of lesion $[F(1,56)=22.9, P<0.0001]$ and a significant lesion $\times$ training-to-lesion interval interaction $[F(1,56)=7.7, P<0.01]$. Planned comparisons $(P<$ 0.05 ) indicated that rats with neurotoxic DH lesions made after training exhibited significantly less freezing than rats in all of the other groups, which did not significantly differ from one another. Thus, neurotoxic DH lesions blocked the expression, but not the acquisition of context fear in rats. The lack of an anterograde deficit in this experiment occurred even though the training-to-testing interval was extended to 1 week for the pretraining groups. Therefore, the lack of deficit in rats with pretraining DH lesions in Experiment 1 cannot be attributed to the use of a short training-to-testing interval.

Collectively, the results from Experiments 1 and 2 indicate that neurons in the $\mathrm{DH}$ are not required to learn fear to contextual stimuli, but are required to express context fear if it was acquired before the $\mathrm{DH}$ lesion was made (neurons in the $\mathrm{DH}$ are not required to express context fear in rats that have been trained following a DH lesion-these rats express context fear normally despite their DH lesion). The results of Experiment 2 provide the first evidence for a role for $\mathrm{DH}$ neurons in the expression of contextual fear conditioning established using unsignaled shock and confirm that neurons in the $\mathrm{DH}$ are not required for the acquisition of context fear. Because the acquisition of contextual fear conditioning established using unsignaled shock is attenuated by electrolytic DH lesions $[14,19,40]$, we suggest that axons passing through the DH in the alveus or fimbria/fornix are critical for the acquisition of context fear.

\section{Experiment 3: Conditional fear following signaled or unsignaled footshock in rats with electrolytic DH lesions}

The results of Experiments 1 and 2 are not consistent with previous studies reporting anterograde deficits in contextual fear conditioning following electrolytic $\mathrm{DH}$ lesions. This inconsistency might be accounted for by either the lesion technique or the behavioral training procedures. With regard to the latter, we have used considerably different conditioning parameters than those used in the studies that have examined the impact of electrolytic DH lesions on fear conditioning. Namely, we have used a smaller number of relatively long (2-s), intense (1.0-mA) footshocks that induce high levels of fear conditioning; Kim et al. [14] used 1-s, 1.0-mA shocks and Phillips and LeDoux [25-27] have used $0.5-\mathrm{s}, 0.5-\mathrm{mA}$ shocks. It is possible that our intense footshocks yield a form of conditional fear that is insensitive to either neurotoxic or electrolytic DH lesions. To test this, we examined the impact of electrolytic DH lesions on the acquisition of contextual fear conditioning using the same signaled and unsignaled shock parameters employed in Experiments 1 and 2. If fear conditioning in our experiments is not sensitive to hippocampal damage, then electrolytic DH lesions, like neurotoxic lesions, should not produce deficits. Alternatively, if the the nature of the lesion accounts for the discrepant results, then electrolytic lesion would be expected to impair fear conditioning generated with our procedures.

\subsection{Materials and methods}

Subjects, surgery and behavioral apparatus. The subjects were 40 adult male Long-Evans rats $(300-500 \mathrm{~g})$ obtained and housed as described in Experiment 1 (Section 2). All surgical procedures were the same as those described for Experiment 1, except that electrolytic lesions were made in the $\mathrm{DH}$ with anodal constant current. Electrodes consisted of Epoxylitecoated stainless steel insect pins (size 00) with insulation scraped from the tips (tip length $=500 \mu \mathrm{m}$ ). Electrolytic DH lesions were made by passing anodal constant current (1.0 mA, $20 \mathrm{~s}$; Grass Instruments, Quincy, MA) at each of the DH coordinates described in Experiment 1 . No electrodes were implanted during sham surgery. The chambers for conditioning, context fear testing and tone fear testing were identical to those described in Experiment 1.

Procedure. The rats were randomly assigned to a $2 \times 2$ (lesion $\times$ training type; $n=10 /$ group) design. For this design, rats received either electrolytic DH lesions or sham surgery 7 days before training with either signaled or unsignaled shocks. All of the procedures for fear conditioning were identical to those described in Experiments 1 and 2. Context and tone fear were assessed as described in Experiment 1, 24 and $48 \mathrm{~h}$ following training, respectively.

Our previous work suggested an interaction between locomotor activity and freezing deficits in rats with hippocampal formation lesions [19]. To examine this further, we compared the effects of electrolytic and excitotoxic DH lesions on locomotor activity measured in the conditioning boxes prior to footshock on the conditioning day. Pre-shock locomotor activity was quantified on the conditioning day by counting the number of times in the 3-min pre-shock period each rat's entire body (minus the tail) crossed from one side of the conditioning chamber to the other. A crossover was counted only if the rat made the response by stepping forward (crossovers were not counted if the rat backed into the other side of the chamber).

Histology and data analysis. Histological verification of lesion location and data analysis were performed as described in Section 2. 


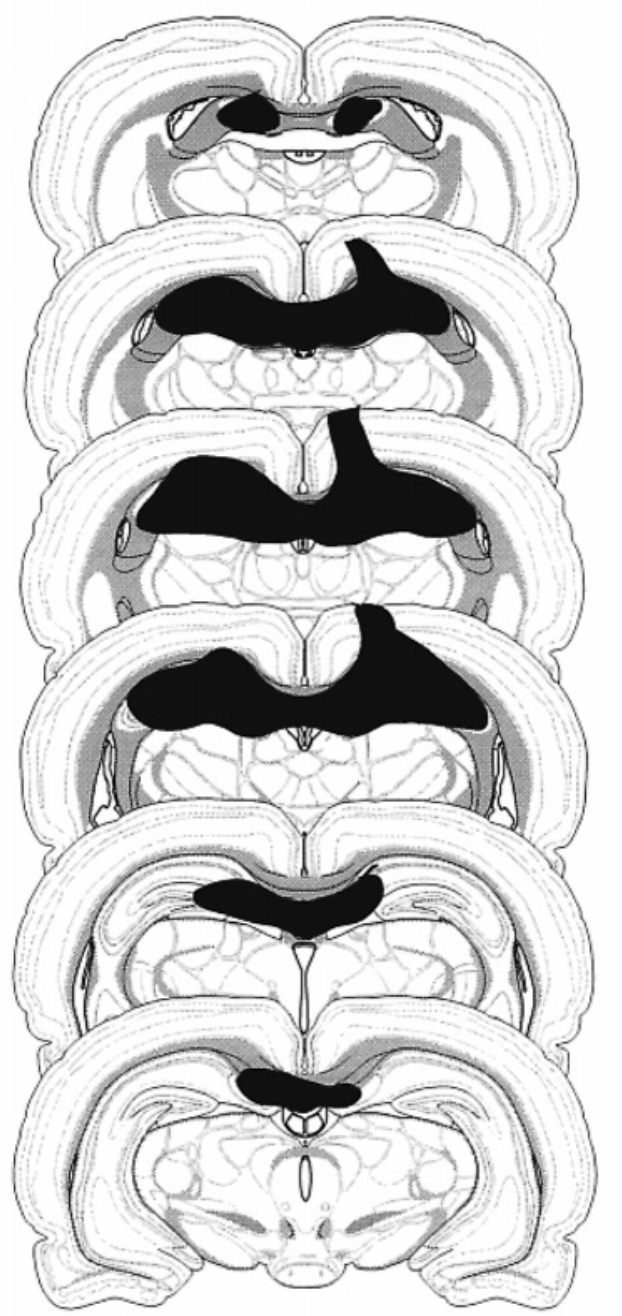

Fig. 6. Schematic representation of a representative electrolytic dorsal hippocampal lesion. Reconstruction of the lesion was made on rat brain atlas templates [35].

\subsection{Results and discussion}

Histology. Electrolytic DH lesions were comparable in size to the neurotoxic DH lesion in Experiments 1 and 2 and comparable to electrolytic lesions in other studies [19]. Thus, the lesions consisted of bilateral damage to hippocampal areas CA1 and CA3, the dentate gyrus and the rostral aspect of the dorsal subiculum. However, unlike neurotoxic lesions, electrolytic lesions damaged axonal tracts (fimbria/fornix, alveus, corpus callosum) in the vicinity of the DH lesion. A reconstruction of a representative electrolytic $\mathrm{DH}$ lesion is shown in Fig. 6.

Behavior. The signaled groups did not differ from the unsignaled groups in any analysis and were therefore collapsed for clarity of presentation. Postshock freezing for the SH and DH groups is shown in Fig. 7(a). A three-way ANOVA with variables of lesion ( $\mathrm{SH}$ and $\mathrm{DH}$ ), training type (signaled and unsignaled) and trial (baseline and one, two and three trials) indicated that, unlike NMDA lesions, electrolytic DH lesions attenuated the acquisition of postshock freezing. This was evident as a significant main effect of lesion $[F(1,34)=$ 6.8, $P<0.02]$ and a significant lesion $\times$ trial interaction $[F(3,102)=2.7, P<0.05]$. Planned comparisons $(P<$ $0.05)$ indicated that rats with $\mathrm{DH}$ lesions exhibited significantly lower freezing than sham rats after the first and second conditioning trials, but not after the third conditioning trial. Thus, rats with DH lesions were able to acquire postshock freezing after three shocks. The $\mathrm{DH}$ lesion-induced deficit was not dependent on the type of training procedure used [lesion $\times$ training type interaction, $F(1,34)=0.02, P=0.88]$. This attenuation of postshock freezing by electrolytic DH lesions is consistent with other reports $[19,40]$.

In addition to attenuating postshock freezing, electrolytic DH lesions produced a significant impairment in contextual freezing during the 8-min extinction test (Fig. 7(b)). A three-way ANOVA with variables of lesion (SH and $\mathrm{DH}$ ), training type (signaled and unsignaled) and 64-s block (eight blocks) revealed a significant main effect of lesion $[F(1,34)=13.2, P<$ 0.001 ] and a nonsignificant lesion $\times$ training type interaction $[F(1,34)=2.4, P=0.13]$. Thus, electrolytic $\mathrm{DH}$ lesions attenuated context freezing independent of the type of conditioning procedure used to train the response. Electrolytic DH lesions also reduced conditional freezing to the tone CS in rats receiving signaled shocks (Fig. 7(c)), although this effect did not reach statistical significance [main effect of lesion, $[F(1,17)=$ 3.6, $P=0.07]$. Nonetheless, it is fair to point out that, as in Experiment 1, this effect would have been detected if more subjects had been included in the experiment. Collectively, these results confirm those from other studies using electrolytic DH lesions [14,19,25,26,40] and indicate that anterograde electrolytic $\mathrm{DH}$ lesions produce significant deficits in the acquisition of both postshock freezing and contextual freezing during extinction testing. Together with the results from Experiments 1 and 2, these results suggest that damage to fibers of passage in the DH is responsible for anterograde deficits in contextual fear conditioning. The basis for the tone freezing deficits rats with DH lesions in the present experiments is not yet understood, but we have observed similar deficits in rats with electrolytic entorhinal cortex lesions [17]. As stated earlier, the tone deficits in rats with DH lesions may consist of a generalization decrement during testing of tone fear.

In some sense, it is not surprising that that neurotoxic DH lesions do not reproduce the effects of electrolytic DH lesions on the acquisition of contextual fear conditioning, because dissociations between the effects of neurotoxic and electrolytic lesions in the hippocampus have been reported in several tasks [9]. For example, electrolytic DH lesions that destroy $20-40 \%$ of the 

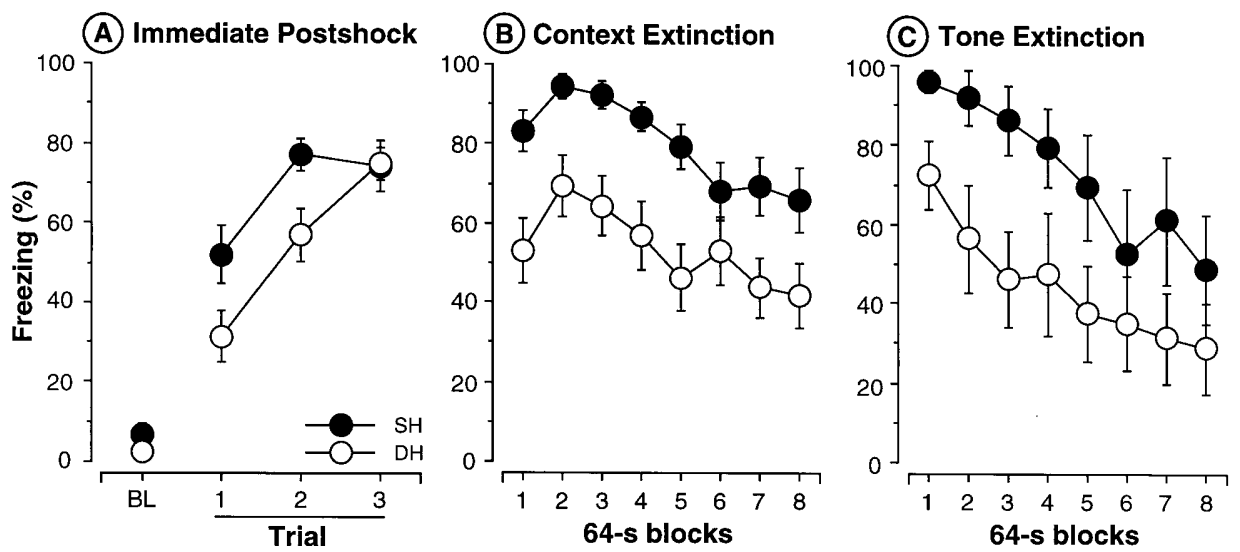

Fig. 7. Pretraining electrolytic lesions of the dorsal hippocampus and conditional freezing. Rats were trained with either signaled or unsignaled shock, but are collapsed in the figure for clarity. (A) Mean ( \pm S.E.M.) percentage of freezing on the conditioning day for rats in the sham (SH, filled circles) and dorsal hippocampus (DH, open circles) groups, (B) mean ( \pm S.E.M.) percentage of freezing during the context extinction test for rats in the sham ( $\mathrm{SH}$, filled circles) and dorsal hippocampus ( $\mathrm{DH}$, open circles) groups and $(\mathrm{C})$ mean $( \pm$ S.E.M.) percentage of freezing during the tone extinction test for rats in the sham ( $\mathrm{SH}$, filled circles) and dorsal hippocampus ( $\mathrm{DH}$, open circles) groups. Only rats receiving signaled training are shown in (C). Anterograde electrolytic DH lesions impaired both postshock freezing and freezing during the context extinction test. Impaired freezing in DH rats during the tone test did not reach statistical significance.

total hippocampal volume produce severe deficits in spatial learning in the Morris water maze [22], whereas neurotoxic DH lesions that destroy the same volume of tissue do not [21]. Similarly, Murray and Mishkin [23] have recently reported that neurotoxic lesions of the hippocampus do not reproduce the visual recognition memory impairments that have been reported following radio frequency hippocampal lesions, which damage fibers of passage [1]. Collectively, these results suggest that damage to axonal fibers of passage in the hippocampus may be responsible, at least in part, for some of the memory deficits reported following electrolytic, aspiration, or radio frequency hippocampal lesions.

We have recently reported a strong correlation between the magnitude of contextual freezing deficits and increased locomotor activity produced by electrolytic fimbria/fornix, DH, or entorhinal cortex lesions [19]. In an effort to understand the basis for the different effects of electrolytic and neurotoxic DH lesions on the acquisition of contextual fear conditioning, we have measured locomotor activity in rats with electrolytic (present experiment) and neurotoxic (Experiment 2) DH lesions. We used cage crossovers during the 3-min preshock period on the conditioning day as the measure of locomotor activity. As shown in Fig. 8, only electrolytic DH lesions produced a significant increase in locomotor activity prior to footshock on the conditioning day. A one-way ANOVA with a variable of group confirmed this observation $[F(2,65)=9.7, P<0.0002]$. Planned comparisons $(P<0.05)$ indicated that the rats in the electrolytic DH (DH-EL) group made significantly more crossovers than rats in the the sham (SH) and excitotoxic DH lesion (DH-EX) groups, which did not differ from one another. Thus, electrolytic and neurotoxic DH lesions have differential effects on both context conditioning and locomotor activity, suggesting that the deficits in the acquisition of context fear and increases in activity in rats with electrolytic DH lesions are related. However, locomotor hyperactivity is unlikely to be the cause of deficits in conditional freezing insofar as there were not significant within-group Pearson correlations between crossovers and freezing $(\mathrm{SH}$ : $r=-0.25, \quad P=0.16$; DH-EX: $r=-0.33, P=0.23$; DH-EL: $r=0.31, P=0.18$ ). Moreover, the correlation

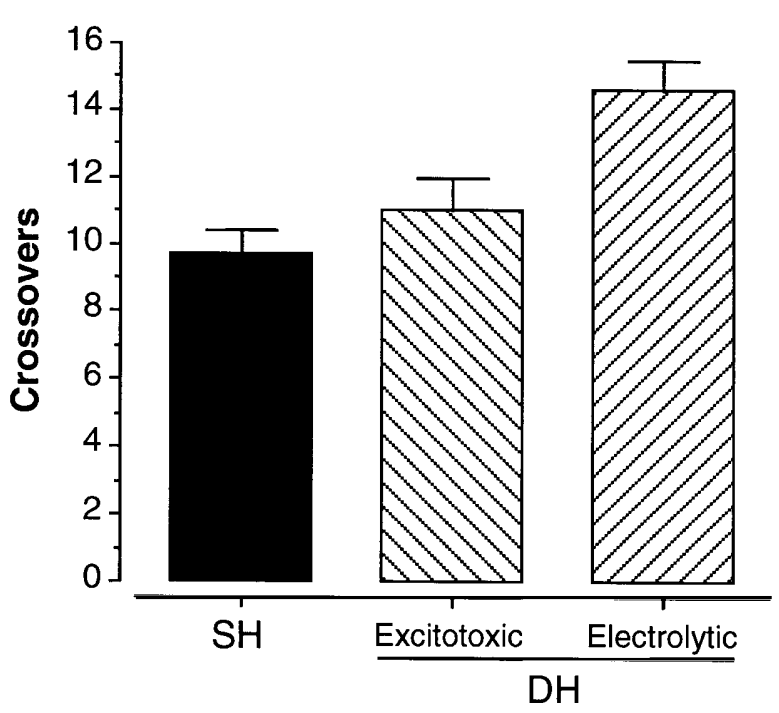

Fig. 8. Neurotoxic and electrolytic lesions of the dorsal hippocampus and pre-shock locomotor activity. Mean ( \pm S.E.M.) crossovers for rats in the SH (filled bar) and DH (hatched bar) groups. Crossovers were scored during the 3-min pre-shock period on the conditioning day, and the data are collapsed across this 3-min interval. Electrolytic, but not excitotoxic lesions of the DH reliably increased crossovers compared to sham surgery. 
between crossovers and freezing was actually positive in the rats with electrolytic DH lesions, which does not account for their increased activity and decreased freezing relative to the sham rats.

\section{General discussion}

The present experiments reveal an interesting pattern of findings. First, neurotoxic DH lesions severely disrupt the expression of fear conditioning when made within one month of training, but have no effect on the acquisition of contextual fear conditioning. They also do not affect short-term memory for contextual fear. Second, electrolytic, but not neurotoxic, DH lesions disrupt the acquisition of context fear. And, third, both electrolytic and neurotoxic DH lesions produce impairments in tone freezing. This pattern of findings was somewhat unanticipated and suggests that a revision of current thinking about hippocampal function in fear conditioning is necessary [13,24] To account for these results, we propose a model with the following features: (1) Rats can acquire context fear using either a configural (hippocampal-dependent) or elemental (hippocampal-independent) strategy; (2) configural strategies normally overshadow elemental strategies; (3) exploration provides both the configural and elemental systems with contextual information; and (4) this exploration is mediated, in part, by fibers of passage in the DH. This model provides a reasonable account of the context conditioning data.

Considering the first two features of the context fear model, we assume that contextual fear conditioning is mediated by different learning strategies subserved by different neural systems. We suggest that intact rats acquire contextual information during fear conditioning using a hippocampal-dependent configural strategy that normally overshadows a nonhippocampal-dependent elemental strategy. Overshadowing, which has been extensively described in both Pavlovian and instrumental tasks (for a review see [33]) describes the phenomenon whereby one stimulus conditions better than another simultaneously presented stimulus. In the present case, we assume that configural representations of context normally overshadow elemental representations of context. For configural learning, the context of the conditioning chamber, which consists of a number of different unimodal stimulus elements (the odor of the chamber, the tactile properties of the grid floor, the spatial dimensions of the chamber, etc.), is assembled into a unique configural representation of 'context'. During training, contextual fear conditioning is established through an association between the configural representation of context and footshock. It has been suggested by others that the formation of configural representations may depend on the integrity of the hippocampal system $[30,33]$. Thus, intact rats that acquire contextual fear conditioning using a configural strategy would be expected to be severely impaired by posttraining neurotoxic DH lesions made before the hippocampus consolidates the configural representation of context.

In addition to this configural strategy rats may be able to use an elemental strategy, that does not require the hippocampus, to acquire contextual fear conditioning. By this view, rats condition fear to a particularly salient unimodal element in the context (e.g. the odor of the chamber) or to several of the less salient contextual elements. In either case, conditioning proceeds without the formation of a unique configural representation of the contextual elements. It follows from this discussion that an elemental strategy would permit normal contextual learning in rats with neurotoxic DH lesions (it also predicts that rats with neurotoxic $\mathrm{DH}$ lesions should condition fear to tones normally). However, this elemental strategy may only be available after a period of recovery from the lesion, because acute, pretraining intra-hippocampal infusions of either muscimol [2] or APV [40] disrupt contextual fear conditioning. Clearly, a more complete understanding of the mechanisms of contextual learning is required to assess the plausibility of this aspect of the model.

The final two features of the model address the different effects of neurotoxic and electrolytic DH lesions on the acquisition of contextual fear conditioning. From the model proposed above, one would predict that rats with electrolytic DH lesions should be able to acquire context fear using an elemental strategy. However, rats with electrolytic DH lesions do not exhibit intact acquisition of contextual fear conditioning. To account for this paradox, we propose that electrolytic $\mathrm{DH}$ lesions disrupt fibers of passage that are importantly involved in both configural and elemental aspects of contextual fear conditioning. More specifically, we propose that electrolytic DH lesions impair context conditioning because they sever projections from the ventral subiculum to the nucleus accumbens [5]. It is suggested that these projections mediate exploratory behavior, which provides both configural and elemental systems with information during learning.

Consistent with this proposal, it has recently been reported that neurotoxic lesions of the nucleus accumbens produce deficits in the acquisition of contextual fear conditioning ([11]; R.F. Westbrook, personal communication). Furthermore, damage to subiculo-accumbens projections may be responsible for locomotor hyperactivity and impaired exploration in rats with electrolytic DH lesions (e.g. [20,36]). As we have seen, rats with neurotoxic DH lesions that spare these axonal projections do not exhibit locomotor hyperactivity (for similar evidence see [37]). Damage to the nucleus accumbens and subiculo-accumbens pathways alters ex- 
ploratory behavior to novel stimuli (e.g. [3]). Therefore, it may be the case that locomotor hyperactivity and impaired exploratory behavior in rats with electrolytic DH lesions prevents them from sampling contextual elements in a normal way. In other words, the sensorymotor interface that normally directs exploratory activity to novel stimuli in intact rats and rats with neurotoxic DH lesions may be disrupted in rats with electrolytic DH lesions, limiting their ability to accumulate information regarding context and consequently impairing contextual fear conditioning.

At this time, the proposed model does not provide a complete account of deficits in tone fear in rats with hippocampal lesions. It is normally assumed that a tone CS is a unitary stimulus that animals process using an elemental strategy. On this basis, deficits following hippocampal lesions would not be predicted. However, we would like to argue that a tone CS is not a unitary stimulus. A tone CS has frequency, amplitude, duration and location, and a given tone activates a specific population of auditory-responsive neurons with different but overlapping tuning curves. Hippocampal lesions would be expected to disrupt any configural contribution to tone conditioning and such configural coding of the tone may only be apparent under certain test conditions. For example, we found that the tone deficit was greatest when the test tone duration exceeded that of the training tone. Thus, the hippocampus may mediate a configural representation of the tone CS that contains information on tone duration. Similarly, Rudy and Pugh [29] have suggested that their is a configural representation of tone CSs that contains specific frequency information, because interfering with configural processing flattened auditory generalization gradients. Certainly, future research is required to specify the conditions that favor configural versus elemental strategies during fear conditioning.

Although this model accounts well for the pattern of deficits we have observed following DH lesions, it also remains possible that this pattern of deficits derives not only from the types of strategies used by rats to acquire context fear, but also from the relatively circumscribed nature of the DH lesions we have used. Thus, rats with neurotoxic DH lesions may use the same strategies as intact rats to acquire context fear, but this learning may be mediated by spared tissue in the ventral hippocampus, subiculum and/or entorhinal cortex. Accordingly, evidence suggests that spatial learning in the Morris water maze proceeds normally in rats with relatively small neurotoxic DH lesions [21]. In fact, neurotoxic $\mathrm{DH}$ lesions must be larger than $40 \%$ of the total hippocampal volume before deficits appear in the Morris water maze [21]. This suggests that spared dorsal and/or ventral hippocampal tissue in rats with small neurotoxic DH lesions is sufficient for spatial learning. Similarly, anterograde memory impairments in mon- keys are relatively mild following circumscribed hippocampal lesions, but increase dramatically with larger lesions [42]. These data suggest that both the locus and size of hippocampal lesions are important variables in determining the severity of anterograde memory impairments. Nonetheless, the fact that circumscribed neurotoxic DH lesions produce severe retrograde deficits in context fear indicates that lesion size is not a critical factor in determining context fear deficits. It is more likely that the involvement of the hippocampus in fear conditioning is determined by the strategy by which fear is acquired during training.

\section{Acknowledgements}

The authors would like to thank Fred Westbrook for providing helpful discussion on the interpretation of the present data. We would also like to thank Fred Helmstetter, Jeansok Kim, Lisa Stefanacci and Stuart Zola for commenting on an earlier draft of the manuscript. This work was supported by a grant from the NIMH (MH37986) to M.S.F. S.M. was supported by an individual NIMH National Research Service Award (MH11061).

\section{References}

[1] P. Alvarez, S. Zola-Morgan, L.R. Squire, L.R. Damage, limited to the hippocampal region produces long-lasting memory impairment in monkeys, J. Neurosci. 15 (1995) 3796-3807.

[2] P.S.F. Bellgowan, F.J. Helmstetter, Effects of muscimol applied to the dorsal hippocampus on the acquisition and expression of cued versus contextual fear conditioning, Soc. Neurosci. Abstr. 21 (1995) 534.

[3] L.H. Burns, L. Annett, A.E. Kelley, B.J. Everitt, T.W. Robbins, Effects of lesions to amygdala, ventral subiculum, medial prefrontal cortex and nucleus accumbens on the reaction to novelty: Implication for limbic-striatal interactions, Behav. Neurosci. 110 (1996) 60-73.

[4] S. Campeau, M. Davis, Involvement of the central nucleus and basolateral complex of the amygdala in fear conditioning measured with fear-potentiated startle in rats trained concurrently with auditory and visual conditioned stimuli, J. Neurosci. 15 (1995) 2301-2311.

[5] N.S. Canteras, L.W. Swanson, Projections of the ventral subiculum to the amygdala, septum and hypothalamus: A PHAL anterograde tract-tracing study in the rat, J. Comp. Neurol. 324 (1992) 180-194.

[6] Y.H. Cho, D. Beracochea, R. Jaffard, Extended temporal gradient for retrograde and anterograde amnesia produced by ibotenate entorhinal cortex lesions in mice, J. Neurosci. 13 (1993) $1759-1766$.

[7] Y.H. Cho, R.P. Kesner, Involvement of entorhinal cortex or parietal cortex in long-term spatial discrimination memory in rats-retrograde-amnesia, Behav. Neurosci. 110 (1996) 436-442.

[8] M.S. Fanselow, Associative vs. topographical accounts of the immediate shock freezing deficit in rats: Implications for the response selection rules governing species specific defensive reactions, Learning Motivation 17 (1986) 16-39. 
[9] J.E. Jarrard, What does the hippocampus really do?, Behav. Brain Res. 71 (1995) 1-10.

[10] L.E. Jarrard, B.S. Meldrum, Selective excitotoxic pathology in the rat hippocampus, Neuropath. Appl. Neurobiol. 19 (1993) 381-389.

[11] M.J. Kiernan, G. Bailey, J. Sims, D. Lukes, J. Cranney, Accumbal lesions attenuate contextual fear conditioning in the rat, Soc. Neurosci. Abstr. 22 (1996) 1381.

[12] J.J. Kim, R.E. Clark, R.F. Thompson, Hippocampectomy impairs the memory of recently, but not remotely, acquired trace eyeblink conditioned responses, Behav. Neurosci. 109 (1995) 195-203.

[13] J.J. Kim, M.S. Fanselow, Modality-specific retrograde amnesia of fear, Science 256 (1992) 675-677.

[14] J.J. Kim, R.A. Rison, M.S. Fanselow, Effects of amygdala, hippocampus and periaqueductal gray lesions on short- and long-term contextual fear, Behav. Neurosci. 107 (1993) 10931098.

[15] J.E. LeDoux, P. Cicchetti, A. Xagoraris, L.M. Romanski, The lateral amygdaloid nucleus: Sensory interface of the amygdala in fear conditioning, J. Neurosci. 10 (1990) 1062-1069.

[16] S. Maren, G. Aharonov, M.S. Fanselow, Retrograde abolition of conditional fear after excitotoxic lesions in the basolateral amygdala of rats: Absence of a temporal gradient, Behav. Neurosci. 110 (1996) 717-718.

[17] S. Maren, M.S. Fanselow, Synaptic plasticity in the basolateral amygdala induced by hippocampal formation stimulation in vivo, J. Neurosci. 15 (1995) 7548-7564.

[18] S. Maren, M.S. Fanselow, The amygdala and fear conditioning: Has the nut been cracked?, Neuron 16 (1996) 237-240.

[19] S. Maren, M.S. Fanselow, Electrolytic lesions of the dorsal hippocampus, fimbria/fornix, or entorhinal cortex produce anterograde deficits in contextual fear conditioning in rats, Neurobiol. Learn. Mem. 67 (1997) 142-149.

[20] G.J. Mogenson, Limbic-motor integration, in: A.N. Epstein, A.R. Morrison (Eds.), Progress in Psychobiology and Physiological Psychology, vol. 12, Academic, London, 1987, pp. 117170.

[21] M.-B. Moser, E.I. Moser, E. Forrest, P. Andersen, R.G.M. Morris, Spatial learning with a minislab in the dorsal hippocampus, Proc. Natl. Acad. Sci. USA 92 (1995) 96979701.

[22] E. Moser, M.-B. Moser, P. Andersen, Spatial learning impairment parallels the magnitude of dorsal hippocampal lesions, but is hardly present following ventral lesions, J. Neurosci. 13 (1993) 3916-3925.

[23] E.A. Murray, M. Mishkin, 40-min visual recognition memory in rhesus monkeys with hippocampal lesions, Soc. Neurosci. Abstr. 22 (1996) 281.

[24] J. O'Keefe, L. Nadel, The Hippocampus as a Cognitive Map, Clarendon, Oxford, 1978.

[25] R.G. Phillips, J.E. LeDoux, Differential contribution of amygdala and hippocampus to cued and contextual fear conditioning, Behav. Neurosci. 106 (1992) 274-285.

[26] R.G. Phillips, J.E. LeDoux, Lesions of the dorsal hippocampal formation interfere with background by not foreground contextual fear conditioning, Learn. Mem. 1 (1994) 34-44.
[27] R.G. Phillips, J.E. LeDoux, Lesions of the fornix but not the entorhinal or perirhinal cortex interfere with contextual fear conditioning, J. Neurosci. 15 (1995) 5308-5315.

[28] N.L. Rempel-Clower, S.M. Zola, L.R. Squire, D.G. Amaral, Three cases of enduring memory impairment after bilateral damage limited to the hippocampal formation, J. Neurosci. 16 (1996) 5233-5255.

[29] J.W. Rudy, C.R. Pugh, A comparison of contextual and generalized auditory-cue fear conditioning: Evidence for similar memory processes, Behav. Neurosci. 110 (1996) 1299-1308.

[30] J.W. Rudy, R.J. Sutherland, Configural association theory and the hippocampal formation: An appraisal and reconfiguration, Hippocampus 5 (1995) 375-389.

[31] N.R. Selden, B.J. Everitt, L.E. Jarrard, T.W. Robbins, Complementary roles for the amygdala and hippocampus in aversive conditioning to explicit and contextual cues, Neuroscience 42 (1991) 335-350.

[32] L.R. Squire, P. Alvarez, Retrograde amnesia and memory consolidation: a neurobiological perspective, Curr. Opin. Neurobiol. 5 (1995) 169-177.

[33] N.S. Sutherland, N.J. Mackintosh, Mechanisms of Animal Discrimination Learning, Academic, New York, 1971.

[34] R.J. Sutherland, J.W. Rudy, Configural association theory: The role of the hippocampal formation in learning, memory and amnesia, Psychobiology 17 (1989) 129-144.

[35] L.W. Swanson, Brain maps: Structure of the Rat Brain, Elsevier, New York, 1992.

[36] I.Q. Whishaw, J.-C. Cassel, M. Majchrzak, S. Cassel, B. Will, 'Short-stops' in rats with fimbria-fornix lesions: Evidence for a change in the mobility gradient, Hippocampus 4 (1994) 577582.

[37] I.Q. Whishaw, L.E. Jarrard, Similarities vs. differences in place learning and circadian activity in rats after fimbria-fornix section or ibotenate removal of hippocampal cells, Hippocampus 5 (1995) 595-604.

[38] K.A. Wiig, L.N. Cooper, M.F. Bear, Temporally graded retrograde-amnesia following separate and combined lesions of the perirhinal cortex and fornix in the rat, Learn. Mem. 3 (1996) $313-325$.

[39] G. Winocur, Anterograde and retrograde amnesia in rats with dorsal hippocampal or dorsomedial thalamic lesions, Behav. Brain Res. 38 (1990) 145-154.

[40] S.L. Young, D. Bohenek, M.S. Fanselow, NMDA processes mediate anterograde amnesia of contextual fear conditioning induced by hippocampal damage: Immunization against amnesia by context preexposure, Behav. Neurosci. 108 (1994) 1929.

[41] S. Zola-Morgan, L.R. Squire, The primate hippocampal formation: Evidence for a time-limited role in memory storage, Science 250 (1990) 288-290.

[42] S. Zola-Morgan, L.R. Squire, S.J. Ramus, Severity of memory impairment in monkeys as a function of locus and extent of damage within the medial temporal lobe memory system, Hippocampus 4 (1994) 1-13. 